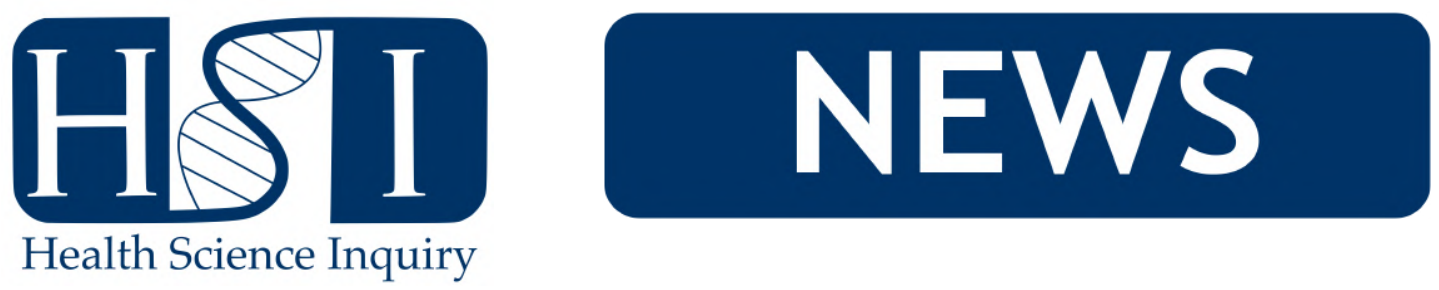

\title{
Emerging Regenerative Therapies for Heart Injury: Addressing an Urgent Need
}

\section{Sarah Shawky}

Cardiovascular disease (CVD) continues to be the leading cause of death worldwide, accounting for over 8.5 million annual mortalities [1]. CVD is also correlated with an increased risk for a heart attack, or myocardial infarction (MI). Following an acute MI, a human adult loses approximately one billion cardiomyocytes - heart muscle cells - which are then replaced by non-contracting fibrous scar tissue $[1,2,3]$. The inability of the damaged myocardium to fully replenish itself weakens the contractile function of the heart in the long-term, further increasing the risk of MI and heart failure over time $[1,4]$. Due to the irreversible nature of cardiac injury, the current gold-standard therapy for advanced heart failure remains cardiac transplantation [1]. However, along with the severely limited supply of suitable donor hearts, the increasing global incidences of myocardial infarction highlight the urgent need for the development of novel, less invasive therapeutic interventions.

Despite substantial advancements in cardiovascular biology and tissue engineering over the last 20 years, no clinicallyavailable means to regenerate damaged cardiac muscle currently exists [5]. However, a variety of technologies to support this approach have been heavily investigated in recent years. These strategies include stem cell-based therapies, which involve the integration of undifferentiated cells into injured host tissue to mediate repair. Another approach involves the generation of biomaterial-based scaffolds, which may support tissue repair by providing a template for regeneration. While several preclinical and clinical studies exploring the use of various types of stem cells have shown promising results in the reversal of cardiac pathologies, others have faced significant barriers $[3,5]$. Some of these obstacles have evoked important research questions, including the need for more efficient methods of stem cell delivery, and the investigation into means of increasing stem cell survival, retention and engraftment within the infarcted heart [2, 3, 6]. Excitingly, cardiac researchers have begun to address these critical questions, providing hopeful answers that may offer a new outlook on the direction of cardiac regenerative medicine.

Dr. Erik Suuronen, a research scientist in the Division of Cardiac Surgery and the director of the Biomaterials Regeneration Program at the University of Ottawa Heart Institute, focuses his research on tissue engineering and cellbased therapeutic approaches for the treatment of cardiac injury. Specifically, he is interested in the development and testing of biomaterials to mobilize and recruit the body's endogenous reparative cells, with aims of creating an environment that better supports and promotes cardiac regeneration. Briefly, Dr. Suuronen describes tissue engineering as a way to direct the repair process - his team is investigating whether a biomaterial scaffold could promote the engraftment of transplanted cells, and hence mediate the regenerative process to reverse cardiac injury. To conduct their research, Dr. Suuronen and his team use various instruments to characterize the physical properties of the materials they design. They also use in vitro and in vivo models to test the ability of these materials to mediate cell function and to promote repair. Specifically, they are interested in studying how the developed biomaterials interact with various cell types to promote regeneration, and how these interactions can be exploited to enhance the efficacy of a potential therapy. Dr. Suuronen and his team recently developed a collagen-based biomaterial which, upon delivery into an injured heart, can preserve its function.

"Upon injection of our collagen-based biomaterial into the infarcted heart, it interacts with multiple cell types to limit chronic inflammation and cell death, reduce adverse remodeling, promote revascularization and improve cardiac function", Dr. Suuronen says.

Using PET and fluorescence imaging techniques, Dr. Suuronen and his team revealed that the injected collagen-based biomaterial is effectively retained and redistributed within murine models of MI [7]. Dr. Suuronen believes that further mechanistic insight into how the injected biomaterial interacts with endogenous cell types to repair the injured heart may reveal key target pathways with novel therapeutic potential. His team is hopeful that biomaterials will soon begin pre-clinical trials to test their safety and efficacy as treatments for cardiac injury - potentially as both a stand-alone therapy and as a vehicle for the delivery of other therapeutic agents.

But in order to develop a physiologically-relevant and clinically-applicable biomaterial therapeutic, Dr. Suuronen says that the final product must be compatible with the human heart. Over the last two years, his team has shifted its 
focus from developing animal protein-derived biomaterials, to utilizing human collagen-based materials instead.

As a final thought, Dr. Suuronen reflects upon the growing ease of finding experts and trainees with the necessary skillset and experience to form the collaborations needed to translate regenerative medicine discoveries into clinical applications. When he first began working in this area of research, it was a challenging endeavour to build the strong, highly-multidisciplinary team necessary - and pivotal - for success. However, nowadays, combining the diverse expertise of scientists, engineers, physicians and imaging specialists is becoming easier, and the field of cardiac regeneration is looking more promising.

Taking an alternate route, Dr. Michael Laflamme, a cardiac cell therapy pioneer at the McEwen Stem Cell Institute, is focused on developing novel therapies for post-MI heart failure based on human pluripotent stem cells (hPSCs) - the only stem cell that can be differentiated into large enough quantities of phenotypically unambiguous cardiomyocytes. Research investigating the remuscularization of the infarcted heart using exogenous cell transplantation began in the early 1990s [8]. Early experiments began by using immature cell types, such as neonatal and fetal cardiomyocytes, skeletal myoblasts and bone marrow-derived hematopoietic cell types $[3,6,8]$. However, due to the ethical issues, efficacy problems and limited generation of de novo cardiac muscle cells associated with the use of each of these cell types, Dr. Laflamme instead focuses his research on another cell type - the infinitely expandable pluripotent stem cell-derived cardiomyocytes.

In addition to having developed methods to guide hPSCs into cardiomyocytes, Dr. Laflamme and his team have shown that when transplanted into models of injured hearts, these cells are capable of electrical coupling with the host myocardium, firing synchronously with the rest of the heart and improving its contractile function. In the mid-2000s, Dr. Laflamme began his work using small animal models, such as rats, and later shifted into the use of guinea pigs and macaques in pioneering work to demonstrate that hPSC-derived cardiomyocytes mediate functional integration and regeneration of the myocardium. Currently, he is focused on surgically delivering a billion of these cells into pig hearts, because of their near-identical size, structure and function to the human heart. Dr. Laflamme anticipates that this is expected to be the last pre-clinical model prior to human pre-clinical trials, which he hopes to initiate in about three to four years.

Dr. Laflamme discusses some of the important obstacles to address prior to considering the translation of this research from in vivo models to humans. This includes overcoming the occurrence of transient arrhythmias following the cell transplantation.

"These [transplanted] cardiomyocytes are unlike those present in an adult; they are less mature and have different electrical properties", Dr. Laflamme explains. "When they are transplanted, transient arrhythmias - abnormal beating rhythms of the heart - are seen in the beginning, while they are "settling" in vivo. Later, these arrhythmias disappear and the cells function effectively once matured. We want to come up with ways to overcome or prevent these arrhythmias - perhaps via antiarrhythmic drugs, or by engineering the cells".

In 2016, the McEwen Centre for Regenerative Medicine at the University Health Network partnered with the new life sciences start-up, BlueRock Therapeutics, driving large-scale research and development in the area of heart muscle regeneration. The manufacturing platform at BlueRock Therapeutics will help to fuel the scalable production of various stem cell types for cellular therapeutics - including pluripotent stem cell-derived cardiomyocytes, which Dr. Laflamme and his team are focused on bringing into the clinic.

Despite the hurdles to be overcome, cardiac regenerative medicine researchers including Drs. Suuronen and Laflamme are excited about the substantial progress that has been made towards the development of novel therapies for cardiac injury. While some laboratories are aggressively exploring strategies to ramp up endogenous cellular regeneration via exogenous biomaterials, others are focused on cellular reprogramming and gene therapy. Overall, the rapid development of these diverse, promising therapies offers hope that their translation from the laboratory to having positive clinical impacts at the bedside is closer than we may have thought.

We would like to extend our thanks to Dr. Erik Suuronen and Dr. Michael Laflamme for sharing their work, and for their time and effort in contributing to this news article.

\section{REFERENCES}

[1] Kochegarov A, Lemanski LF. New trends in heart regeneration: a review. Journal of stem cells \& regenerative medicine. 2016;12(2):61.

[2] Lin Z, Pu WT. Strategies for cardiac regeneration and repair. Science translational medicine. 2014;6(239):239rv1239 rv1.

[3] Romagnuolo R, Laflamme MA. Programming cells for cardiac repair. Current opinion in biotechnology. 2017;47:4350.

[4] Bertero A, Murry CE. Hallmarks of cardiac regeneration. Nature Reviews Cardiology. 2018;p. 1. 
[5] Duelen R, Sampaolesi M. Stem cell technology in cardiac regeneration: a pluripotent stem cell promise. EBioMedicine. 2017;16:30-40.

[6] Bilgimol JC, Ragupathi S, Vengadassalapathy L, Senthil NS, Selvakumar K, Ganesan M, et al. Stem cells: An eventual treatment option for heart diseases. World journal of stem cells. 2015;7(8):1118.

[7] Ahmadi A, Thorn SL, Alarcon EI, Kordos M, Padavan DT, Hadizad T, et al. PET imaging of a collagen matrix reveals its effective injection and targeted retention in a mouse model of myocardial infarction. Biomaterials. 2015;49:18-26.

[8] Lundy SD, Gantz JA, Pagan CM, Filice D, Laflamme MA. Pluripotent stem cell derived cardiomyocytes for cardiac repair. Current treatment options in cardiovascular medicine. 2014;16(7):319.

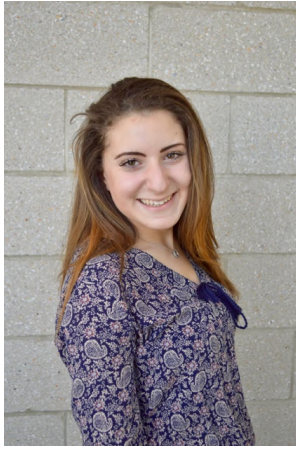

Sarah received her Honours Bachelor of Health Sciences degree from McMaster University, where she specialized in Biomedical Discovery \& Commercialization. Passionate about the intersection between scientific discoveries and their translation into clinical medicine, Sarah is enthusiastic about contributing to innovative research. She is currently an MSc candidate at the Leslie Dan Faculty of Pharmacy, University of Toronto, and is a recipient of the CIHR Canada Graduate Scholarship. Sarah's MSc research is focused on studying the role of activated nuclear hormone receptors in the prevention of atherosclerosis. 